\title{
The Serological Survey for Human Cysticercosis Prevalence in Mbulu District, Tanzania
}

\author{
Beda J. Mwang'onde ${ }^{1,2^{*}}$, Gamba Nkwengulila ${ }^{1}$, Mwita Chacha ${ }^{1}$ \\ ${ }^{1}$ Department of Zoology and Wildlife Conservation, University of Dar es Salaam, Dar es Salaam, Tanzania; ${ }^{2}$ Division of Livestock \\ and Human Disease Vector Control, Tropical Pesticides Research Institute, Arusha, Tanzania. \\ Email: *bedajohnm@gmail.com
}

Received July $4^{\text {th }}, 2012$; revised August $2^{\text {nd }}, 2012$; accepted September $3^{\text {rd }}, 2012$

\begin{abstract}
Human cysticercosis, a zoonotic disease due to Taeniasolium, is of the highly debilitating and stigmatizing neglected tropical diseases due to epilepsy, ophthalmia and dermatological disorders in endemic developing countries. Tanzania is among the sub-Saharan African countries with an average prevalence of porcine cysticercosis 17.2\% [1], which increases the risk of human cysticercosis infection. Studies on people with epilepsy in northern zone of Tanzania show a $16.2 \%$ [2] to be suffering from neurocysticercosis. While this was the case, there were no single report on the prevalence of human cysticercosis neither to regular hospital visitors, admissions, and nor general public that result to insufficient deployment of intervention strategies. Study was performed to assess the prevalence of human cysticercosis in general public in Mbulu district. The cephalic venous blood was collected from assorted community members. Serum was extracted and then subjected to Cysticercus IgG Western Blot Assay for human cysticercosis sero-screening. It was found that about $16.3 \%$ of the community members had antibodies signifying infection by human cysticercosis. This is the first ever study to assess the prevalence of human cysticercosis in the country and it has revealed the problem to be very high. This study can be valuable for deployment of appropriate intervention measures on human cysticercosis in the study area and extend to the entire country.
\end{abstract}

Keywords: Human Cysticercosis; Taeniasolium; Mbulu-Tanzania; Prevalence

\section{Introduction}

Human cysticercosis is a zoonotic disease which is caused by the larval stage of pork tapeworm (Taeniasolium cysticercosis) and poses serious public health consequences in developing countries [3]. The disease affects no less than 20 million people with an annual fatality rate of approximately 50,000 people worldwide [4]. Human cysticercosis accounts for about $10 \%$ of all acute neurological admissions and a main cause of late onset epilepsy in endemic areas [5] with about $20 \%-50 \%$ of all late-onset epilepsy cases globally [6]. Human cysticercosis infection can go unnoticed therefore, its public health consequence may be grave than it is estimated. Recent trends due to international tourism into remote or rural areas, expansion of global business and increase of the number of trans-migrants from rural to urban areas as well as increase of immigrants and refugees, have drastically increased the cases of taeniasis and cysticercosis in developed countries, Orthodox Jewish [7] and Muslim communities [8,9].

Human cysticercosis is highly reported in Latin Amer-

\footnotetext{
"Corresponding author.
}

ica, Asia and Africa. Studies in some regions of Mexico show a prevalence of $3.6 \%$ of the general population [4]; in Asia 3.2\% [10]; in Caribbean particularly in Haiti 2.8\%, [11] and; in western Africa the prevalence of human cysticercosis range from $1.3 \%-2.4 \%$ [12]. On the other hand, in sub-Saharan Africa about 30\% of people with epilepsy have been diagnosed with neurocysticercosis [13]. Neurocysticercosis due to Taeniasolium cysticercosis is reported to be a disease of poverty and under development as to why being one of the main causes of epilepsy in developing countries [14]. Nevertheless, the importation T. solium by tourism, migration of tapeworm carriers or indirectly by importations of infected pigs, or pig meat poses risks of the disease in Europe [15]. Because of globalization, human cysticercosis begins to appear in industrialized countries particularly Portugal and Northern Spain [16] and in North America[17]; and in Islamic [18] and Orthodox Jewish [17] communities. That is to say that the epidemiology of human cysticercosis spares no community in the world because of globalization.

The risk of human cysticercosis in Tanzania is very high because the average prevalence of Taenia cellulosae was 
reported to be $17.2 \%$ [1]. On the other hand, piggery industry has significantly grown in the country due to rapid economic turnover and a drastic rise in pork consumption in many rural and urban areas [19]. In urban communities, pork is a delicacy that goes together with alcohol consumption [20]. The free range system of keeping pigs, lack and or improper use of latrines, clandestine slaughtering and marketing of pork, absence of pork inspection and barbecuing [21-23], further increase the risk of human cysticercosis infection in Tanzania populations'. With the reported high risks of human cysticercosis, studies mainly have been reporting porcine cysticercosis $[1,6,21$, 24]. The few studies that report cysticercosis infections in human are based on neuro cysticercosis particularly to people with epilepsy [2,25]. Hospital based studies in people with epilepsy in northern Tanzania report an average neurocysticercosis prevalence of $16.2 \%$ [2,25]. Because human cysticercosis can go unnoticed for quite long, these reported studies in human do not adequately provide with the cysticercosis prevalence picture in the Tanzania's population.

This study reports a serological survey of human cysticercosis based on antibodies determination in the general community from Mbulu district, in the northern part of Tanzania.

\section{Materials and Methods}

\subsection{Study Area and Ethical Clearance}

The study was carried out in Mbulu District $\left(3.80^{\circ} \mathrm{S}\right.$ $4.50^{\circ} \mathrm{S}, 35.00^{\circ} \mathrm{E}-36.00^{\circ} \mathrm{E}$ ). Mbulu district has a population of 321,359 (DED, 2011). The area lies at an estimated altitude of 1000 - $2400 \mathrm{~m}$ above sea level. Climate ranges from semi-arid to sub-humid with an annual rainfall of $<400$ and $>1200 \mathrm{~mm}$, respectively. There are two rainy seasons with two peaks of maximum rains. The long rainy season extends from about March to mid- May and the short rainy period from November to December. In between (January through February) is a dry season. Relative humidity ranges from $55 \%$ to $75 \%$ and mean annual temperatures range from $15^{\circ} \mathrm{C}$ to $24^{\circ} \mathrm{C}$. The ethical clearance and approval of the study was sought from the National Institute for Medical Research (NIMR) (ref. NIMR/HQ/R.8a/Vol. IX/1297). NIMR is one of the United Republic of Tanzania research organs responsible for health research and the key board that oversees all regulatory issues of health research in the country.

\subsection{Data Collection for Serological Survey of Human Cysticercosis}

The goal of this survey was to assess the proportion of people in the study area carrying Abs of $T$. solium cysticercosis as an indication of infection by the cestode. Blood samples from randomly selected and consented community members were collected.

About 544 individuals of different age groups and sex from general community participated in the study. About 3 mls of cephalic venous blood was collected from each of the consented participants by the use of evacuated collection tubes. The samples were centrifuged at 3500 rpm at Mbulu District Hospital for serum extraction, aliquoted and stored in cryogenic vials in freezers before they were transported to the University of Dar es Salaam for further storage at $-20^{\circ} \mathrm{C}$. The determination of antibodies for human cysticercosis infection was carried out by using Cysticercus IgG Western Blot Assay (LDBIO Diagnostics 69009 Lyon-France) at Biotechnology Laboratory, Department of Molecular Biology and Biotechnology, University of Dar es Salaam.

Cysticercus IgG Western Blot Assay detects the presence of cysticercosis antibodies. However, the test may be cross-reactive with other parasites. The principle of the test is based on Cysticercus antigens from a crude larval extract which have been resolved by electrophoresis into bands and transferred by electroblotting onto a nitrocellulose membrane which is readly cut into testing strips. The presence on the strip of a minimum of 2 welldefined bands among the 6 (i.e. 6 - 8; 12; 23 - 26; 39; 45; 50 - $55 \mathrm{kDa}$ ) described bands were considered as an indication of human cysticercosis. However, the presence of the lower molecular weight band (6 - $8 \mathrm{kDa}$ ) was also described as an indication of active cysticercosis [26].

\subsection{Statistical Analysis}

Data entry and validation was carried out using ms-excel 2010 version (Ms Corp., Redmond, WA, USA). Data on human cysticercosis seroprevalencewas analyzed using the Statistical Package for Social Sciences version 19.0 (SPSS Statistic 19, 2010 IBM). Descriptive statistics were used to obtain the percentage prevalence of both human cysticercosis and other helminthes infections that were able to be detected by the kit in different age groups of the sampled population. The unequal variance $t$-test (Welch's $t$-test) was used to test for the variations of infection between different age groups and gender, $\propto=0.05$.

\section{Results}

\subsection{Prevalence Rate of Human Cysticercosis and other Helminthes}

The mean age of respondents was $34.49 \pm 18.44$ with a range of 92 . The female to male ratio was 0.64 . The prevalence of human cysticercosis for circulating cysticercal antibodies in Mbulu general community was $16.3 \%$ ( $n=544)$. The infection rate was found to increase with age and the male participants were more 
positive to human cysticercosis than female participants. Based on age cohorts, the prevalence rates were $2 \%$ (0 10 years); $8 \%$ (11 - 15 years); and 52\% (16 - 45 years). Then the prevalence rate decreased to $19 \%$ (45 - 60 years); $5 \%$ (61 - 75 years); and $1 \%$ in above 76 years of age. About $75 \%$ of the positive cases were males with $56.3 \%$ of all positive cases in both female and males suggesting neurocysticercosis infection. The frequencies of other parasitic infections were echinococcosis $12.1 \%$, schistosomiasis $6.8 \%$, toxocariasis $14.1 \%$, and toxoplasmosis $1.7 \%$. The age cohort between 16 - 45 years was significantly more infected with human cysticercosis than cohorts between $0-10$ years $(\mathrm{p}<0.00015, \mathrm{df}=46)$; 11 15 years $(\mathrm{p}<0.0065$, df $=51) ; 46-60$ years $(\mathrm{p}<0.0062$, $\mathrm{df}=61) ; 61-75$ years $(\mathrm{p}<0.0004$, df $=48)$; and 76 and above $(\mathrm{p}<0.0001$, $\mathrm{df}=45)$.

\section{Discussions}

Human cysticercosis infections are one of the major causes of epilepsy and other neurological syndromes which are highly debilitating and thus resulting into social stigmatization in developing endemic countries. Based on the findings from this study, the disease is highly prevalent in Mbulu. Because Mbulu district is one of the main sources of pork in urban, the risk of human infection in Tanzania mighty be very high as under cooked pork consumption increase chances of pork tapeworm infestation. The fact that the epidemiology of human cysticercosis is favoured by food, water or environmental contamination by pork tapeworm eggs from tapeworm carriers, the threat of the disease in the country might be holistic.

The study found anticysticercal antibodies for human cysticercosis in eighty eight participants corresponding to $16.3 \%$ of all tested community members from the general public in Mbulu, a district in the northern zone of Tanzania. These findings correspond to the $16.7 \%$ (by AgELISA) of active human cysticercosis which was reported from Mbozidistrict [27], a district in a southern zone of Tanzania, again one of the main sources of pork in both rural and urban areas. Elsewhere, in Rwanda and Mozambique, the seroprevalence of human cysticercosis was found to be $2.8 \%$ and $12.1 \%$ [28,29], respectively. While all human cysticercosis results contrasted in here are from Africa south of Sahara, the observed differences might have been contributed by target groups for study, method of testing and risk factors for the disease. The risk of human cysticercosis in Mbulu is very high because of traditional behaviour of keeping pigs under free range system.

On the other hand, the observed prevalence is significantly less to that reported byNsengunya et al. [30] which was $31.5 \%(n=648)$ of the health individuals positive to anticysticercal antibodies in Burundi. The hu- man cysticercosis prevalence reported by this study had the highest frequency in ages between 16 - 45 years. This is in complement with that reported by Samir et al. [31], however, under the reported study the higher frequency is attributed by high return of the participants for the reported age range than other cohorts. Males were detected more positive (75\%) to human cysticercosis than females $(\mathrm{p}=0.0099$, $\mathrm{df}=86)$. The differences in human cysticercosis seropositivity rates between sexes and age groups possibly reflect levels of exposure by gender to risk factors. Males and the reported productive age group (16 45) might be spending more time in local brews clubs where they are subjected to eating undercooked pork and or contaminated food or drinks by tapeworm eggs. However, further studies need to be done to find out whether there is any genetic, sexual or senility relationship for human cysticercosis infections. The prevalence rate of human cysticercosis was also found to decrease with the increasing age particularly at ages above 50 years old. This may have been also contributed by the low return rate to participating to the study because of decreased chances of being picked for sampling as the life expectancy in Tanzania is 48 years due to HIV/AIDS pandemic and the leading cause of death, and malaria, the number one killer of children under five [32]. High prevalence of human cysticercosis in the study region is contributed by the free range system of keeping pigs and the indiscriminate disposal of human faeces that are later easily picked by pigs and thus enhance the parasite lifecycle. The best intervention measure for this highly debilitating and stigmatizing disease is education to deny human faeces-pig contact followed by mass chemotherapy by physician prescribed anthelmintic drug of choice to humans.

Based on these findings, human cysticercosis is a big problem in the country that requires an in-depth review and serological survey all over the country, mapping and then development of a logical framework for intervention strategies of the disease.

\section{Acknowledgements}

The research for this paper was carried out within the framework of the consortium Afrique One "Ecosystem and Population Health: Expanding Frontiers in Health". Afrique One is funded by the Wellcome Trust (WT087535MA) and the University of Dar es Salaam for hosting and experimental facilitation of the project.

\section{REFERENCES}

[1] I. K. Phiri, H. Ngowi, S. Afonso, E. Matenga, M. Boa, S. Mukaratirwa, S. Githigia, M. Saimo, C. Sikasunge, N. Maingi, et al., "The Emergence of Taenia Solium Cysticercosis in Eastern and Southern Africa as a Serious Ag- 
ricultural Problem and Public Health Risk,” Acta Tropica, Vol. 87, No. 1, 2003, pp. 13-23.

[2] A. S. Winkler, J. Blocher, H. Auerd, T. Gotwalde, W. Matuja and E. Schmutzhard, "Anticysticercal and Antitoxocaral Antibodies in People with Epilepsy in Rural Tanzania," Transactions of the Royal Society of Tropical Medicine and Hygiene, Vol. 102, No. 10, 2008, pp. 10321038.

[3] N. Praet, K. Kanobana, C. Kabwe, V. Maketa, P. Lukanu, P. Lutumba, K. Polman, P. Matondo, N. Speybroeck et al, "Taenia Solium Cysticercosis in the Democratic Republic of Congo: How Does Pork Trade Affect the Transmission of the Parasite?” PLOS Neglected Tropical Diseases, Vol. 4, No. 9, 2010, p. e817. doi:10.1371/journal.pntd.0000817

[4] R. W. Tolan, “Taenia Infection,” 2011. http://wwwtheinfectiousdiseasesdailyom/

[5] F. Fregni, “Taenia Solium (Taeniasis/Cysticercosis),” Collaborative Course on Infectious Diseases, 2008.

[6] H. A. Ngowi, I. K. Phiri, S. Afonso, E. Matenga, M. E. Boa, S. Mukaratirwa, S. Githigia, M. Saimo, C. Sikasunge, N. Maingi, et al., "Taenia Solium Cysticercosis in Eastern and Southern Africa: An Emerging Problem in Agriculture and Public Health," Southeast Asian Journal of Tropical Medicine and Public Health, Vol. 35, No. 1, 2004, pp. 266-270.

[7] F. Sorvillo, P. Wilkins, S. Shafir and M. Eberhard, "Public Health Implications of Cysticercosis Acquired in the United States," Emerging Infectious Diseases, Vol. 17, No. 1, 2011, pp. 1-6.

[8] K. Swastika, C. I. Dewiyani, T. Yanagida, Y. Sako, M. Sudarmaja, P. Sutisna, T. Wandra, N. S. Dharmawan, K. Nakaya, M. Okamoto, et al., "An Ocular Cysticercosis in Bali, Indonesia Caused by Taenia Solium Asian Genotype,” Parasitology International, Vol. 61, No. 2, 2012, pp. 378-380. doi:10.1016/j.parint.2011.11.004

[9] I. Francis, P. R. Hira, N. A. Abdella, R. Gupta, F. M. AlAli, S. Grover, N. Khalid, S. Abdeen, J. Iqbal, M. Wilson and V. C. Tsang, "Cysticercosis: Imported and Autochthonous Infections in Kuwait," Transactions of the Royal Society of Tropical Medicine and Hygiene, Vol. 98, No. 4, 2004, pp. 233-239. doi:10.1016/S0035-9203(03)00061-0

[10] W. C. Cao, P. C. Van Der, C. L. Gao, J. F. Xu, X. C. Cao, Z. H. Cui, Z. X. Ren and J. D. F. Habbema, "Seroprevalence and Risk Factors of Human Cysticercosis in a Community of Shandong, China," Southeast Asian Journal of Tropical Medicine and Public Health, Vol. 27, No. 2, 1996, pp. 279-285.

[11] C. P. Raccurt, P. Agnamey, J. Boncy, J.-H. Henrys and A. Totet, "Seroprevalence of Human Taenia Solium Cysticercosis in Haiti,” Journal of Helminthology, Vol. 83, No. 2, 2009, pp. 113-116.

[12] N. A. Mafojanea, C. C. Appletonb, R. C. Krecekc, L. M. Michaeld and A. L. Willingham III, "The Current Status of Neurocysticercosis in Eastern and Southern Africa," Acta Tropica, Vol. 87, No. 1, 2003, pp. 25-33.

[13] A. L. Willingham III and A. Mugarura, "Taenia Solium Tapeworms and Epilepsy in Uganda,” International Epilepsy News, Vol. 1, 2008, pp. 10-12.
[14] D. K. Pal, A. Carpio and J. W. A. S. Sander, "Neurocysticercosis and Epilepsy in Developing Countries," Journal of Neurology, Neurosurgery and Psychiatry, Vol. 68, No. 2, 2000, pp. 137-143.

[15] European Commission, "Opinion of the Scientific Committee on Veterinary Measures Relating to Public Health on the Control of Taeniosis/Cysticercosis in Man and Animals," Health \& Consumer Protection Directorate General, Brussels, 2000.

[16] N. M. Canas, S. L. Calado and J. Vale, "Treatment of Racemose Neurocysticercosis of the Spine,” Revista de Neurologia, Vol. 40, No. 9, 2005, pp. 544-547.

[17] P. M. Schantz, P. P. Wilkins and V. C. W. Tsang, "Immigrants, Imaging, and Immunoblots: The Emergence of Neurocysticercosis as a Significant Public Health Problem," In: W. M. Scheld, W. A. Craig and J. M. Hughes, Eds., Emerging Infections 2, ASM Press, Washington DC, 1998, pp. 213-242.

[18] W. X. Shandera and J. S. Kass, "Neurocysticercosis: Current Knowledge and Advances," Current Neurology and Neuroscience Reports, Vol. 6, No. 6, 2006, pp. 453-459. doi:10.1007/s11910-006-0046-3

[19] M. V. Johansen, "Draft Action Plan for Prevention and Control of Taenia Solium Cysticercosis/Taeniosis in Eastern and Southern African Region,” Cysticercosis Working Group in Eastern and Southern Africa, Morogoro, 2008.

[20] A. Secka, T. Marcotty, R. De Deken, E. V. Marck and S. Geerts, "Porcine: Cysticercosis and Risk Factors in the Gambia and Senegal," Journal of Parasitology Research, Vol. 2010, 2010, Article ID: 823892.

[21] M. E. Boa, E. A. Mahundi, A. A. Kassuku, A. L. Willingham III and N. C. Kyvsgaard, "Epidemiological Survey of Swine Cysticercosis Using Ante-Mortem and PostMortem Examination Tests in the Southern Highlands of Tanzania,” Veterinary Parasitology, Vol. 139, No. 1-3, 2006, pp. 249-255. doi:10.1016/j.vetpar.2006.02.012

[22] H. A. Ngowi, A. A. Kassuku, G. E. Maeda, M. E. Boa, H. Carabin and A. L. Willingham, "Risk Factors for the Prevalence of Porcine Cysticercosis in Mbulu District, Tanzania,” Veterinary Parasitology, Vol. 120, No. 4, 2004, pp. 275-283.

[23] F. P. Lekule and N. C. Kyvsgaard, "Improving Pig Husbandry in Tropical Resource-Poor Communities and Its Potential to Reduce Risk of Porcine Cysticercosis," Acta Tropica, Vol. 87, No. 1, 2003, pp. 111-117. doi:10.1016/S0001-706X(03)00026-3

[24] E. M. Mkupasi, H. A. Ngowi and H. E. Nonga, "Prevalence of Extra-Intestinal Porcine Helminth Infections and Assessment of Sanitary Conditions of Pig Slaughter Slabs in Dares Salaam City, Tanzania,” Tropical Animal Health and Production, Vol. 43, No. 2, 2010, pp. 417-423. doi:10.1007/s11250-010-9708-X

[25] A. L. Willingham, L. J. Harrison, E. M. Fèvre and M. E. Parkhouse, "Inaugural Meeting of the Cysticercosis Working Group in Europe,” Emerging Infectious Diseases, Vol. 14, No. 12, 2008. doi:10.3201/eid1412.080889

[26] C. Simac, P. Michel, A .Andriantsimahavandy, P. Esterre and A. Michault, "Use of Enzyme-Linked Immunosorbent Assay and Enzyme-Linked Immunoelectrotransfer 
Blot for the Diagnosis and Monitoring of Neurocysticercosis,” Parasitology Research, Vol. 81, No. 2, 1995, pp. 132-136. doi:10.1007/BF00931618

[27] G. Mwanjali, "Prevalence and Practices Associated with Human Taenia Solium Infection in Mbozi District, Mbeya Region,” Dissertation, Muhimbili University of Health and Allied Sciences (MUHAS), Dar es Salaam, 2010.

[28] E. Newell, F. Vyungimana, S. Geerts, I. Van Kerckhoven, V. C. W. Tsang and D. Engels, "Prevalence of Cysticercosis in Epileptics and Members of Their Families in Burundi," Transactions of the Royal Society of Tropical Medicine and Hygiene, Vol. 91, No. 4, 1997, pp. 389-391. doi:10.1016/S0035-9203(97)90251-0

[29] A. Zoli, O. Shey-Njila, E. Assana, J.-P. Nguekam, P. Dorny, J. Brandt and S. Geerts, "Regional Status, Epidemiology and Impact of Taenia Solium Cysticercosis in Western and Central Africa,” Acta Tropica, Vol. 87, No. 1, 2003, pp. 35-42. doi:10.1016/S0001-706X(03)00053-6

[30] G. Nsengiyumva, M. Druet-Cabanac, B. Ramanankandrasana, B. Bouteille, L. Nsizabira and P. M. Preux, "Cysticercosis as a Major Risk Factor for Epilepsy in Burundi, East Africa,” Epilepsia, Vol. 44, No. 7, 2003, pp. 950955.

[31] S. H. Vora, D. D. Motghare, A. M. Ferreira, M. S. Kulkan and S. F. Vaz, "High Prevalence of Human Cysticercosis in a Rural Village in Western India,” Tropical Medicine and Health, Vol. 36, No. 3, 2008, pp. 137-138.

[32] URT, “Tanzania country profile,” 2012. http://www.usaid.gov/ 